\title{
Effective Resistance of Gromov-Hyperbolic Graphs: Application to Asymptotic Sensor Network Problems
}

\author{
Edmond A. Jonckheere \& Mingji Lou and João Hespanha \& Prabir Barooah
}

\begin{abstract}
The technique of effective resistance has seen growing popularity in problems ranging from escape probability of random walks on graphs to asymptotic space localization in sensor networks. The results obtained thus far deal with such problems on Euclidean lattices, on which their asymptotic nature already reveals that the crucial issue is the large scale behavior of such lattices. Here we investigate how such results have to be amended on a class of graphs, referred to as Gromov hyperbolic, which behave in the large scale as negatively curved Riemannian manifolds. It is argued that Gromov hyperbolic graphs occur quite naturally in many situations. Among the results developed here, we will mention the nonvanishing probability of escape of a random walk to a Cantor set Gromov boundary and the facts that the space localization error of sensors networked in a Gromov hyperbolic fashion grows linearly with the distance to a sensor whose geographical position is known, but would become uniformly bounded in an idealized situation in which the geographical locations of the nodes at the Gromov boundary are known
\end{abstract}

\section{INTRODUCTION}

The effective resistance between two nodes in a graph is the voltage drop that would be observed in a electrical network obtained by placing one resistor at each edge of the graph, when a unit current is injected in one of the nodes and removed from the other. In this paper, we extend the result of Doyle and Snell [12] on the effective resistance of a regular tree of degree bounded but greater than 2 and its ramifications in various asymptotic space localization and coordination problems for autonomous agents as initiated by Barooah and Hespanha [5], [3], [4].

A tree is regular if the degree of its nodes, except for the root, is constant. Under this degree condition, the effective resistance from the root of the tree to "infinity" is finite. The latter has the important consequence that a random walk on the tree has a finite probability of escaping to infinity. In the context of computer security, it follows that a worm is more likely to escape to infinity and therefore cause more damage than one that is recurrent (see Remark 1). In the context of localization based on relative measurements, this means that if only the absolute positions of the far-away leafs of a large tree are available, all nodes in the tree can still be accurately localized just based on noisy relative measurements between adjacent nodes in the graph [4]. The reader is referred to [5] for other connections between effective resistance and distributed control problems.

These authors are with the Dept. of Elec. Eng., Univ. of South. California, Los Angeles, CA 90089, jonckhee@usc.edu.

These authors are with the Univ. of California, Santa Barbara, CA 93106, hespanha@ece.ucsb.edu
The natural generalization of the concept of tree is that of Gromov hyperbolic graph [14]. Intuitively, a Gromov hyperbolic graph is a graph that has the property that it looks like a tree when viewed at a distance, where the concept of "viewing at a distance" is formalized in large-scale geometry, also referred to as coarse geometry or asymptotic metric theory [15]. The importance of Gromov hyperbolic graphs is that scale free, and hence Internet, graphs have that property [18], [23], [19]. Since Gromov hyperbolic graphs are a generalization of trees, the natural question is, "What is the effective resistance of a Gromov hyperbolic graph?" The importance of effective resistance of Gromov hyperbolic graphs stems from the above mentioned fact that effective resistances arise naturally in a variety of propagation and localization problems.

Before extending the result of Doyle and Snell, a technical question is whether such concept as "effective resistance between the root and infinity" can be defined for those hyperbolic graphs, in which the tree structure might not be completely obvious. A "root" of a hyperbolic graph is a socalled quasi-pole [10]. A quasi-pole $\Omega$ is a compact subset of an infinite graph $G$ such that for every vertex $v$ of $G$ there exists a geodesic ray emanating from $\Omega$ and passing within a bounded distance of $v$. One of the premises of coarse geometry is that finite subsets, like $\Omega$, can be coalesced to points. As such, $\Omega$ can be coalesced to $\omega \in \Omega$, which becomes the "root" of the graph. Once a root is chosen, the next issue is to define "infinity" so as to be able to define the effective resistance between the quasi-pole and infinity. In the context of the coarse geometry of Gromov hyperbolic graphs, "infinity" is the Gromov boundary at infinity of the graphs, $\partial_{\infty} G$. The latter is defined as the equivalence class of infinite geodesic rays emanating from the quasi-pole under the equivalence relation that two rays are equivalent if their Hausdorff distance remains finite [9, III.H.3].

While the Gromov boundary $\partial_{\infty} G$ of a regular tree can be easily visualized, this is not so when all that is known about $G$ is that it is Gromov hyperbolic, since it could be anything ranging from $\mathbb{N}$ to the circle $S^{1}$, passing by the Cantor set, the Menger curve, etc. (See [21] for a survey.) The difficulty is that the topology of $\partial_{\infty} G$ dictates such application-relevant issues as the Cheeger isoperimetric constant and the exponential growth of balls [10]. As far as the present paper is concerned, in order to have finite effective resistance between $\Omega$ and $\partial_{\infty} G, \partial_{\infty} G$ must have the cardinality of the continuum.

Since in this paper we use coarse geometric techniques in which we do not care about distortion as long as it is 
uniformly bounded, the exact value of the effective resistance $R\left(\omega, \partial_{\infty} G\right)$ is irrelevant. Is relevant, however, the question of whether the effective resistance is vanishing, finite $(0<$ $\left.R\left(\omega, \partial_{\infty} G\right)<\infty\right)$, or infinite. In fact, the latter are coarse geometry invariants.

Due to space limitations, most proofs have been omitted, but they are available in [20].

\section{MATHEMATICAL SET-UP}

\section{A. Graphs}

A graph $G$ is defined by its vertex set and its edge set, $(V, E)$. The edge with end vertices $u, v$ will be written $u v$. All graphs here have bounded local geometry, i.e., the degree of their nodes is uniformly bounded. As such, an infinite graph has infinitely many vertices. Edges are equipped with a length function, $\ell: E \rightarrow \mathbb{R}^{+}$, with the property that

$$
\ell_{\min }:=\inf _{e \in E} \ell(e)>0, \quad \ell_{\max }:=\sup _{e \in E} \ell(e)<\infty
$$

Upon identification of every edge with a homeomorph of the unit interval $[0,1]$, the length function is easily extended to Lebesgue-measurable subsets of the edges.

A path from $x$ to $y$ is a continuous map $p:[a, b] \rightarrow G$ such that $p(a)=x, p(b)=y$. The length of a path joining $x$ to $y$ is the sum of the lengths of the (subsets of) edges traversed by the path. The distance $d(x, y)$ between $x, y$ is the infimum of the lengths of all paths joining $x$ to $y$. The latter will sometimes be referred to as length distance. With this length distance, $(G, d)$ becomes a metric space.

An isometric embedding $f: G \rightarrow H$ of the graph $G=$ $\left(V_{G}, E_{G}\right)$ into the graph $H=\left(V_{H}, E_{H}\right)$ is a map (usually induced by a vertex transformation $V_{G} \rightarrow V_{H}$ ) that preserves the metric, viz., $d_{H}(f(x), f(y))=d_{G}(x, y)$.

A geodesic $\gamma=[x y]$ from $x$ to $y$ in $G$ is an isometric embedding $\gamma:[0, \ell(\gamma)] \rightarrow G$ such that $\gamma(0)=x, \gamma(\ell(\gamma))=$ $y$. Because of the bounded local geometry, every pair of points $x, y$ can be joined by a geodesic $[x y]$ such that $\ell([x y])=d(x, y)$. In such a situation, $(G, d)$ is said to be a geodesic metric space.

\section{B. Gromov hyperbolicity}

A geodesic triangle $\triangle u v w$ on vertices $u, v, w$ is defined as $[u v] \cup[v w] \cup[w u]$. A graph $(G, d)$, or an arbitrary geodesic metric space $(X, d)$ for that matter, is Gromov hyperbolic if there exists a $\delta_{s}<\infty$ such that, $\forall \triangle u v w$, we have $[v w] \subset N_{\delta_{s}}([u v]) \cup N_{\delta_{s}}([u w])$, where $N_{\delta_{s}}([u v])$ denotes the $\delta_{s}$-neighborhood of $[u v]$ for the metric $d$. An equivalent characterization is the existence of a constant $\delta_{i}<\infty$ such that, $\forall \triangle u v w$, for points $i(v, w) \in[v w], i(u, w) \in$ $[u w], i(u, v) \in[u v]$ such that $d(u, i(u, v))=d(u, i(u, w))$, $d(v, i(v, w))=d(v, i(v, u)), d(w, i(w, v))=d(w, i(w, u))$, we have $\operatorname{diam}\{i(u, v), i(v, w), i(w, u)\} \leq \delta_{i}$. It can be shown that $\delta_{s}<\infty$ iff $\delta_{i}<\infty$ (see [9, Chap. III.H, Prop. 1.17]). Yet a third equivalent definition states that, in any quadrilateral, the largest sum of the lengths of pairs of opposite diagonals cannot exceed the medium sum by more than $2 \delta_{q}<\infty$ (see [9, p. 411]).
The most extreme example of a Gromov hyperbolic graph is the tree, as $\delta_{i}=\delta_{s}=\delta_{q}=0$.

The following theorem formulates an important property of Gromov hyperbolic spaces, a property that is sometimes even taken to be the definition of Gromov-hyperbolicity [10, Def. 1.1]:

Theorem 1: Let $(X, d)$ be a $\delta_{s}$-Gromov hyperbolic space. Let $\gamma_{1}, \gamma_{2}$ be two arc length parameterized geodesics such that $\gamma_{1}(0)=\gamma_{2}(0)=v^{0}$. Assume that, for some $R>0$, $d\left(\gamma_{1}(R), \gamma_{2}(R)\right)>3 \delta_{s}$. Then any path $p$ joining $\gamma_{1}(R+r)$, $\gamma_{2}(r+R)$ and outside the ball $B_{R+r}\left(v^{0}\right)$ is such that

$$
\ell(p) \geq \delta_{s} 2^{\frac{r}{\delta_{s}}-2}
$$

This theorem has the (negative) consequence that, if an outage affects a ball $B_{R+r}\left(v^{0}\right)$, a message scheduled to transit along $[u v] \ni v^{0}$ will have to make a detour of exponential length $\delta_{s} 2^{\frac{r}{\delta_{s}}-2}$ to circumvent the outage. As shown in [14], this bound can be refined to $\delta_{s} 2^{\frac{r+R}{\delta_{s}}-1}$.

\section{Quasi-isometry}

A $(\lambda, \epsilon)$-quasi-isometric embedding of the graph $G=$ $\left(V_{G}, E_{G}\right)$ into the graph $H=\left(V_{H}, E_{H}\right)$ is a (not necessarily continuous) function $f: G \rightarrow H$ (usually induced by a vertex transformation $V_{G} \rightarrow V_{H}$ ) such that

$$
\frac{1}{\lambda} d_{G}(x, y)-\epsilon \leq d_{H}(f(x), f(y)) \leq \lambda d_{G}(x, y)+\epsilon,
$$

$\forall x, y \in G$. Such an $f$ is quasi-injective in the sense that

$$
f(x)=f(y) \quad \Rightarrow \quad d_{G}(x, y) \leq \epsilon \lambda, \quad \forall x, y \in G .
$$

$f$ is said to be a quasi-isometry if, in addition, there exists a constant $c$ such that

$$
d_{H}(f(G), z) \leq c, \quad \forall z \in H
$$

which means that $f$ is quasi-surjective. In this case, $f$ has at least one quasi-inverse $f^{-q}: H \rightarrow G$ in the sense that there exists a constant $d$ for which

$$
\begin{aligned}
& d_{G}\left(f^{-q} \circ f(x), x\right) \leq d, \quad \forall x \in G, \\
& d_{H}\left(f \circ f^{-q}(z), z\right) \leq d, \quad \forall z \in H,
\end{aligned}
$$

and this quasi-inverse is both quasi-injective and quasisurjective. Quasi-isometry is an equivalence relation.

\section{Gromov boundary}

A geodesic ray $r$ emanating from $\omega \in V$ is an isometric embedding $r:[0, \infty) \rightarrow G$ such that $r(0)=\omega$. The same ray will sometimes be written as $r(0) / r(\infty)$. Two rays $r, r^{\prime}$ are said to be equivalent iff $\sup _{s \in[0, \infty)} d\left(r(s), r^{\prime}(s)\right)<\infty$; equivalently, if $d_{\mathcal{H}}\left(r, r^{\prime}\right)<\infty$, where $d_{\mathcal{H}}$ denotes the Hausdorff distance. The set of equivalence class of rays is the Gromov boundary, $\partial_{\infty} G$. The equivalence class of the ray $r$ is written $r_{\infty}$. Given two rays, $r_{\infty}^{u}, r_{\infty}^{w} \in \partial_{\infty} G$, parameterized by the arc length $s$, we define

$$
\rho_{\infty}\left(r_{\infty}^{u}, r_{\infty}^{w}\right):=\lim \inf _{s \rightarrow \infty} e^{-\epsilon\left(s-\frac{1}{2} d_{G}\left(r^{u}(s), r^{w}(s)\right)\right)}
$$

The above is a semimetric [6, I.1.5] on $\partial_{\infty} G$. The idea behind this definition is that, in a tree-like graph, two rays 
$r^{u}, r^{w}$ emanating from the same quasi-pole $\omega$ will first follow the same path and then separate at some vertex $a\left(r^{u}, r^{w}\right)$, the "common ancestor" of the points $r^{u}(\infty), r^{w}(\infty)$. Then $\rho_{\infty}\left(r_{\infty}^{u}, r_{\infty}^{w}\right)=e^{-\epsilon d\left(\omega, a\left(r^{u}, r^{w}\right)\right)}$. Another intuition behind $\rho_{\infty}$ is as follows: Write the hyperbolic law of cosines in $\triangle \omega r^{u}(s) r^{w}(s)$ as embedded in a surface of small negative sectional curvature $\kappa=-\epsilon^{2}$ :

$$
\begin{aligned}
\cosh \epsilon d_{G} & \left(r^{u}(s), r^{w}(s)\right)= \\
& \cosh \epsilon d\left(\omega, r^{u}(s)\right) \cosh \epsilon d\left(\omega, r^{w}(s)\right) \\
& \quad-\sinh \epsilon d\left(\omega, r^{u}(s)\right) \sinh \epsilon d\left(\omega, r^{w}(s)\right) \cos \hat{\omega}
\end{aligned}
$$

where $\hat{\omega}$ is a short for $\angle r^{u}(s) \omega r^{w}(s)$. Since the arc length parameterization of geodesic rays is an isometry, $d\left(\omega, r^{u}(s)\right)=d\left(\omega, r^{w}(s)\right)=s$. Then, for a distance $d$ such that $\epsilon d \gg 1, \cosh \epsilon d \approx e^{\epsilon d}$ and, for a small angle $\hat{\omega}$, $\cos \hat{\omega} \approx 1-\frac{\hat{\omega}^{2}}{2}$. With these approximations, the hyperbolic law of cosines yields

$$
e^{\epsilon d_{G}\left(r^{u}(s), r^{w}(s)\right)}=1+e^{2 \epsilon s} \frac{\hat{\omega}}{2}
$$

In light of the above, $\rho_{\infty}$ turns up to be the angle metric

$$
\rho_{\infty}\left(r_{\infty}^{u}, r_{\infty}^{w}\right)=\lim \inf _{s \rightarrow \infty} \frac{\angle r^{u}(s) \hat{\omega} r^{w}(s)}{\sqrt{2}}
$$

Now we make the semimetric $\rho_{\infty}$ a metric by enforcing the triangle inequality

$$
d_{\infty}\left(r_{\infty}^{u}, r_{\infty}^{w}\right):=\inf _{r^{u}=a^{0}, a^{1}, \ldots, a^{n}=r^{w}} \sum_{i=0}^{n-1} \rho_{\infty}\left(a^{i}, a^{i+1}\right)
$$

For $\epsilon<\log 2 / \delta_{G}, d_{\infty}$ is a visual metric in the sense that

$$
\frac{1}{4} \rho_{\infty}\left(r_{\infty}^{u}, r_{\infty}^{w}\right) \leq d_{\infty}\left(r_{\infty}^{u}, r_{\infty}^{w}\right) \leq \rho_{\infty}\left(r_{\infty}^{u}, r_{\infty}^{w}\right)
$$

(See [16, Sec. 2], [9, III.H.3.19].) This distance in turn defines a topology on $\partial_{\infty} G$.

The Gromov boundary might have varying cardinalities, and even Gromov boundaries with the same cardinality might be quite different. An example of $\operatorname{card}\left(\partial_{\infty} G\right)=$ $\aleph_{0}$ is provided by Figure 1 . It should also be obvious that the cardinality of the Gromov boundary of a binary tree $T_{2}$ is $2^{\aleph_{0}}$, which is the cardinality of the continuum $c$. Another example of $\operatorname{card}\left(\partial_{\infty} G\right)=c$ is provided by $G=\operatorname{Cay}\left(\pi_{1}\left(M^{n}\right)\right)$, the Cayley graph of the fundamental group of a compact Riemannian manifold $M^{n}$ of curvature uniformly bounded from above by $\kappa<0$ (see [11, V.58]). The significant difference between the continua $\partial_{\infty} T_{2}$ and $\partial_{\infty} \operatorname{Cay}\left(\pi_{1}\left(M^{n}\right)\right)$ is that the former is a Cantor set while the latter is $S^{n-1}$.

Other examples of Gromov boundaries that are circles include the Poincaré disk or any of its tessellation by the image of a fundamental domain [22, 4.10].

Theorem 2: Under a quasi-isometry $f: G \rightarrow H, G=$ $\left(V_{G}, E_{G}\right)$ is Gromov hyperbolic iff $H=\left(V_{H}, E_{H}\right)$ is Gromov hyperbolic; furthermore, $f$ induces a (quasi-Möbius) homeomorphism $f_{\infty}: \partial_{\infty} G \rightarrow \partial_{\infty} H$.

It transpires form the above that, for a Gromov hyperbolic graph to be quasi-isometric to a tree, it is necessary that its Gromov boundary be a Cantor set.

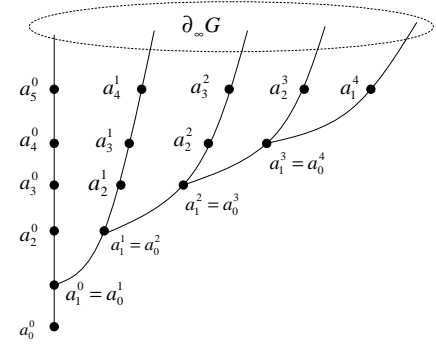

Fig. 1. A tree, the Gromov boundary of which has cardinality $\aleph_{0}$.

\section{E. Isoperimetric behavior and exponential growth of balls}

Given an infinite graph $G$, the combinatorial Cheeger isoperimetric constant is defined as

$$
h(G)=\inf _{S \subseteq V_{G}} \frac{|\partial S|}{|S|}
$$

where the infimum is over all finite subsets, $|S|$ denotes the cardinality, and

$$
\partial S=\{v \in V \backslash S: v \in N(s) \text { for some } s \in S\} .
$$

Observe that $\partial S$ can be interpreted as the boundary of $S$ and therefore if $h(G)>0$ the above means that the "volume" $|S|$ of a subset of vertices is bounded by a linear function of the "area" $|\partial S|$ of its boundary.

The exponential growth of balls is clearly related to the condition $h(G)>0$. Indeed, if a subset $S$ of vertices is "infected" at time $t=0$, the number of vertices infected prior to or at time $t \geq 0$ is bounded from below by $|S|(1+h(G))^{t}$. It turns out that a Gromov hyperbolic graph need not have $h(G)>0$. For the latter to hold, an extra condition on the boundary at infinity is needed:

Theorem 3: Let $G$ be a infinite, bounded geometry, complete, Gromov hyperbolic graph with a quasi-pole. If, in addition, the infimum of the diameters of the connected components of $\partial_{\infty} G$ is $>0$, then $h(G)>0$, which implies that $\lambda_{\min }(L(G))>0$.

There is a slight refinement of the preceding result:

Theorem 4: $\lambda_{\min }(L(G)) \geq \frac{h^{2}(G)}{4}$.

\section{INSTANCES OF COARSE GEOMETRY}

Here we make the preceding concepts a little more pallatable by linking them with known engineering concepts.

\section{A. Instances of hyperbolic graphs}

Gromov hyperbolic graphs are an idealization of situations that readily occur in practice. Typically, we are given a set of agents $\left\{v^{i}\right\}=: V$, which attempt to form an ad hoc network, and which in doing so define a distance function $d: V \times V \rightarrow \mathbb{R}^{+}$. Typically, the distance $d\left(v^{i}, v^{j}\right)$ is the communication delay between $v^{i}$ and $v^{j}$. As such, $(V, d)$ becomes a metric space. The question is to determine the manifold $M^{n}$ and its Riemannian metric $g$ such that the agents can be thought of as operating on the manifold; precisely, the question is whether 
there exists an isometric embedding $(V, d) \hookrightarrow\left(M^{n}, g\right)$. Specifically, there exists an isometric embedding $(V, d) \hookrightarrow$ $\left(\mathbb{H}^{n}, g\right)$, where $\mathbb{H}^{n}$ denotes the standard $n$-dimensional Riemannian manifold of constant sectional curvature $\kappa<0$, iff $\operatorname{det}\left\{\cosh \left(d\left(v^{i}, v^{j}\right) \sqrt{-\kappa}\right)\right\}_{\{1, \ldots, k\} \times\{1, \ldots, k\}}=(-1)^{k+1}$. (See [6], [7].) Practically speaking, we cite a few specific situations that naturally lead to negatively curved networks.

a) Growth/preferential attachment graphs: They have been shown to be scaled Gromov hyperbolic [18], [19].

b) High power transceivers in a wireless sensor network: They have a tendency to network in a negatively curved graph, while the low power transceivers rather network in a positively curved graph [2].

c) Geometric optics propagation reflecting on convex obstacles: Assume that in the two-dimensional Euclidean space $\mathbb{R}^{2}$, we define a set of transceivers $\left\{v^{i}\right\}$ along with convex obstacles. Assume that, at least for some pairs of agents, communication can only be established by radio waves reflecting on convex obstacles. It can be shown that the embedding is in a negatively curved surface of a genus equal to the number of convex obstacles.

d) Delaunay triangulation of nonuniformly distributed vertices: Assume a set of agents $\left\{v^{i}\right\}$ are nonuniformly distributed in $\mathbb{R}^{2}$. The Delaunay triangulation $(V, E)$ of a set of Gauss distributed agents is shown in Fig. 2 and is negatively curved in the following sense. Define the clustering coefficient as cluster $\left(v^{i}\right)=\frac{\#\left\{\left(v^{i} v^{j}, v^{i} v^{k}\right): v^{j} v^{k} \in E\right\}}{\left(\begin{array}{c}\operatorname{deg}\left(v^{i}\right) \\ 2\end{array}\right)} \in$ $[0,1]$. When $0 \leq \operatorname{cluster}\left(v^{i}\right) \leq \frac{1}{2}$, the curvature is locally negative [2], [13]. The distribution of cluster is shown in Fig. 3, indicating negative curvature.

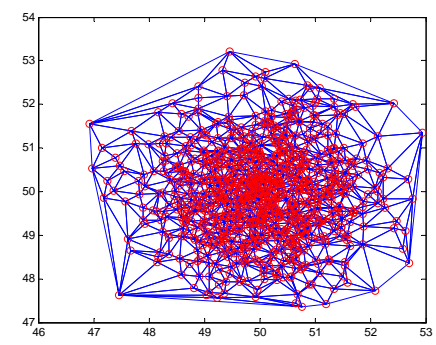

Fig. 2. Delaunay triangulation of nonuniformly distributed agents.

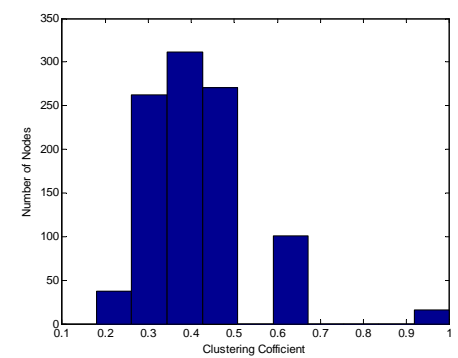

Fig. 3. Clustering coefficient distribution of Delaunay triangulation.

\section{B. Instances of quasi-isometries}

The examples are plentiful, the most obvious being that a Euclidean lattice is quasi-isometric to the Euclidean space of the same dimension. Precisely, the natural embedding $\mathbb{Z}^{n} \rightarrow$ $\mathbb{R}^{n}$ is a $(\lambda=1, \epsilon=1)$-quasi-isometry. More generally, we have the following:

Theorem 5: The drawing function $f: G \rightarrow \mathbb{E}^{n}$ of a graph $G$ that is both sparse and dense in the $n$-dimensional Euclidean space $\mathbb{E}^{n}$ is a $(\lambda, \epsilon)$-quasi-isometry for finite constants $\lambda, \epsilon>0$.

\section{EFFECTIVE RESISTANCE: A GENERALIZED RAYLEIGH MONOTONICITY PRINCIPLE}

The graph $G=(V, E)$ can be made into an electrical network by replacing every edge by a resistor of resistance $R_{e}=\ell(e)$. If $\mathbf{j}\left(v^{i}\right)$ denotes the current injected at node $v^{i}$, and $v\left(v^{i}\right)$ denotes the voltage at node $v^{i}$ relative to some ground potential, then $j=L v$, where $L$ is the Laplacian operator defined as $(L v)_{i}=\sum_{v^{j} \in N\left(v^{i}\right)} \frac{\left(v_{i}-v_{j}\right)}{R_{e\left(v^{i}, v^{j}\right)}}$, where $N\left(v^{i}\right):=\left\{v \in V: v v^{i} \in E\right\}$ and $R_{e\left(v^{i}, v^{j}\right)}$ is the resistance of the edge $v^{i} v^{j}$. If $\ell(e)=R_{e}=1, \forall e \in E$, then $L=\operatorname{diag}\left(\operatorname{deg}\left(v^{i}\right)\right)-A$, where $A$ is the adjacency matrix. It is easily verified that $L \geq 0$. To define the effective resistance at a driving point vertex $v^{i}$, a subset $V_{0}$ of shortcircuited vertices, connected to the graph, is declared at ground potential, $v\left(V_{0}\right)=0$. If $V_{0}$ is finite and at finite distance from some driving point vertex $v^{i} \notin V_{0}$, the effective resistance is defined as $R\left(v^{i}, V_{0}\right)=\left(L_{0}^{-1}\right)_{i i}$, where $L_{0}$ is the operator obtained after removing the rows and columns of $L$ corresponding to $V_{0}$, easily seen to be invertible. If the graph is infinite, viewing $L: \ell^{2}(\mathbb{N}) \rightarrow \ell^{2}(\mathbb{N})$ as a bounded Hilbert space operator requires $v\left(\partial_{\infty} G\right)=0$, so that the 0 reference potential is naturally set across $\partial_{\infty} G$. By the same token, boundedness of $L$ requires $i\left(v^{i}\right)=0, \forall v^{i} \in \partial_{\infty} G$. Assuming for a moment that $\lambda_{1}(L)>0$, where $\lambda_{1}(L)=\inf \operatorname{spec}(L)$, then $\left(L^{-1}\right)_{i i}$ is the effective resistance $R\left(v^{i}, \partial_{\infty} G\right)$ between $v^{i}$ and $\partial_{\infty} G$. The latter consideration makes $\lambda_{1}(L)>0$ a crucial issue:

Theorem 6: $R\left(v^{i}, \partial_{\infty} G\right)<\bar{R}<\infty, \forall v^{i} \in V_{G}$ iff $\lambda_{1}(L(G))>0$.

One can also show that effective resistance satisfies the following triangle inequality

$$
R_{G}(u, w) \leq R_{G}(u, v)+R_{G}(v, w), \quad \forall u, v, w \in V_{G} .
$$

Hence effective resistance is a distance, which is different from the length distance, unless $G$ is a tree.

The following monotonicity result can be viewed as a generalization of Rayleigh's Monotonicity Law [12] to "quasiembeddings":

Theorem 7 (Rayleigh Monotonicity Principle): Consider two uniformly-bounded degree graphs $G=\left(V_{G}, E_{G}\right)$, $H=\left(V_{H}, E_{H}\right)$ for which there exists a quasi-injective function $f: G \rightarrow H$ and constants $\lambda, \epsilon<\infty$ such that

$$
d_{H}(f(x), f(y)) \leq \lambda d_{G}(x, y)+\epsilon, \quad \forall x, y \in G .
$$


Then there exists a finite constant $\alpha>0$ such that

$$
R_{H}(f(x), f(y)) \leq \alpha R_{G}(x, y), \quad \forall x, y \in V_{G} .
$$

If, in addition, $f$ is quasi-surjective then we also have that

$$
R_{H}(z, w) \leq \alpha R_{G}\left(f^{-q}(z), f^{-q}(w)\right)+\beta, \forall z, w \in V_{H},
$$

where $f^{-q}(\cdot)$ is a quasi-inverse of $f$ and $\beta$ a finite constant. When $f$ is actually surjective, then (6) holds with $\beta=0$. In the expressions above, $R_{G}$ and $R_{H}$ denote effective resistances in electrical networks constructed from the graphs $G$ and $H$, respectively.

The following result can be obtained by applying Theorem 7 to a quasi-isometry $f$ and to its quasi-inverse $f^{-q}$.

Corollary 1: Suppose that $f$ is a $(\lambda, \epsilon)$-quasi-isometry between two uniformly-bounded degree graphs $G=\left(V_{G}, E_{G}\right)$, $H=\left(V_{H}, E_{H}\right)$. Then there exist finite constants $\alpha, \beta>0$ such that

$$
\frac{1}{\alpha} R_{G}(x, y)-\beta \leq R_{H}(f(x), f(y)) \leq \alpha R_{G}(x, y),
$$

$\forall x, y \in V_{G}$, where $R_{G}$ and $R_{H}$ denote effective resistances in electrical networks constructed from the graphs $G$ and $H$, respectively. When $f$ is actually injective, then (7) holds with $\beta=0$.

\section{TWO ASYMPTOTIC PROBLEMS}

Here we formulate two asymptotic problems amenable to effective resistance methods:

1) The escape probability of a random walk on a Gromovhyperbolic graph, closely related to the effective resistance between the root and the Gromov boundary of the graph.

2) The asymptotically space localization error at large distance from the reference sensor, closely related to the asymptotic effective resistance between two points in the limit of large distance.

\section{A. Escape probability of random walks}

The exponential growth of balls is relevant to a propagation scheme in which every node infects every neighboring node. Other processes include random walks on a graphthat is, processes through which an infected node infects only one of its neighbors chosen at random. More specifically, if the walker is at $v^{i}$, the probability of jumping to $v^{j} \in N\left(v^{i}\right)$ is

$$
p\left(v^{i}, v^{j}\right)=\frac{\frac{1}{R_{e\left(v^{i}, v^{j}\right)}}}{\sum_{v \in N\left(v^{i}\right) \frac{1}{R_{e\left(v, v^{j}\right)}}}}
$$

The problem is to determine whether there is a nonvanishing probability of the walk reaching the Gromov boundary at infinity. The latter is called escape probability. A slight generalization of $[12$, Sec. 1.3.4] yields

$$
p \operatorname{esc}\left(\omega, \partial_{\infty} G\right)=\frac{\frac{1}{R\left(\omega, \partial_{\infty} G\right)}}{\sum_{v^{i} \in N(\omega)} \frac{1}{R_{e\left(\omega, v^{i}\right)}}}
$$

where $\omega$ is a vertex of a quasi-pole. In other words, the probability of escape from a vertex of a quasi-pole to the boundary at infinity is proportional to the reciprocal of the effective resistance between $\omega$ and the Gromov boundary. As already said, the mere fact that the graph is Gromov hyperbolic with a quasi-pole does not provide enough data to determine an exact value for $R\left(\omega, \partial_{\infty} G\right)$, but it is enough to determine whether or not $R\left(\omega, \partial_{\infty} G\right)$ is finite.

Remark 1. The difference between a randomly walking worm escaping to infinity and an exponentially growing percolating worm is a matter of the gap between $\lambda_{1}(L(G))$ and $h^{2}(G) / 4$, as specified by Theorem 4 .

\section{B. Space localization}

Assume that autonomous agents distributed in the Euclidean space $\mathbb{E}^{2}$ or $\mathbb{E}^{3}$ communicate via the graph $G$. Assume that a subset of vertices $V_{0}$ has known geographical position and that the position of the other agents has to be computed from noisy measurement of the distances $d_{G}\left(v^{i}, v^{j}\right), v^{i} v^{j} \in$ $E$, and azimuth data. The following is proved in [4]:

Theorem 8: If $V_{0}=\left\{v^{0}\right\}$, then the unbiased least square estimation error of the geographical position $x\left(v^{i}\right)$ of $v^{i}$ is given by

$$
E\left(\left\|x\left(v^{i}\right)-\hat{x}\left(v^{i}\right)\right\|^{2}\right)=R_{G}\left(v^{i}, v^{0}\right) .
$$

If $G$ is Gromov hyperbolic, and under the idealized situation where $V_{0}=\partial_{\infty} G$, we have

$$
E\left(\left\|x\left(v^{i}\right)-\hat{x}\left(v^{i}\right)\right\|^{2}\right)=R\left(v^{i}, \partial_{\infty} G\right) .
$$

\section{RESULTS}

The solution to the two problems defined in the previous section relies on a generic theorem, stating that a Gromov hyperbolic graph is quasi-isometric to a tree. Several such results are already known (see, e.g., [8]), but here we need to set-up the quasi-isometry in a specific way that easily translates to effective resistance and geographic estimation errors.

Theorem 9: A planar Gromov hyperbolic graph $G$ with a quasi-pole $\Omega$ and a Cantor Gromov boundary is (quasisurjectively) quasi-isometric to a tree $T$. Furthermore, if $\ell(e)=1, \forall e \in E$, the quasi-isometry can be taken such that

$$
d_{G}(u, v)-\delta_{i} \leq d_{T}(u, v) \leq \lambda d_{G}(u, v), \quad \forall u, v \in V
$$

with $\lambda=7 \delta_{i}+2$.

We note that, in [8], it is shown that there exists a quasiisometric embedding from a binary tree to a Gromov hyperbolic graph with Cantor Gromov boundary. The problem is that this embedding is not in general quasi-surjective and hence cannot be used to construct a quasi-isometry between the graph and the binary tree.

\section{A. Escape probability}

Theorem 10: Let $G$ be a bounded geometry, Gromov hyperbolic graph with a quasi-pole $\Omega$ and a Gromov boundary that is a Cantor set. Then $0<R\left(\omega, \partial_{\infty} G\right)<\infty$, i.e., there is a nonvanishing probability of escape from the quasi-pole to the boundary at infinity $\partial_{\infty} G$. 
We observe that in [8] a quasi-isometric embedding $f$ : $T \rightarrow G$ is set up from a binary tree $T$ to a Gromov hyperbolic graph $G$. This immediately leads to $R_{G}(u, v) \leq$ $R_{T}(u, v)$ and the upper bound $R_{G}\left(\omega, \partial_{\infty} G\right)<\infty$. As the preceding shows, the difficulty is the lower bound.

As an illustration, consider a regular tree with node degree 3. In this case, the Gromov boundary is the collection of all paths from the root to infinity. Since on the way from the root to $\partial_{\infty} G$, each edge gives birth to two other edges, the number of paths from the root to $\partial_{\infty}$, hence the cardinality of $\partial_{\infty} G$, is $2^{\aleph_{0}}$, the cardinality of the continuum. It also follows that the paths can be binary encoded, the same way as the Cantor set is.

To provide a counterexample that $\aleph_{0}$, the cardinality of $\mathbb{N}$, is not enough to guarantee $R\left(\omega, \partial_{\infty} G\right)<\infty$, consider the following tree construction: Let $A^{0}=a_{0}^{0} a_{1}^{0} a_{2}^{0} \ldots$ be a semi-infinite string of vertices such that $d\left(a_{i}^{0}, a_{j+1}^{0}\right)=1$. We attach, at $a_{1}^{0} \in A^{0}$, a replica $A^{1}$ of $A^{0}$. Specifically, $a_{1}^{0}=a_{0}^{1}$. Then we attach at $a_{1}^{1} \in A^{1}$ another replica $A^{2}$. Specifically, $a_{1}^{1}=a_{0}^{2}$. From here on, we iterate by attaching further replica $A^{k}, k \geq 3$, such that $a_{1}^{k}=a_{0}^{k+1}$. Clearly, the cardinality of $\partial_{\infty} G$ is $\aleph_{0}$. As far as the resistance is concerned, $R\left(a_{0}^{0}, \partial_{\infty} G\right)=1+R\left(a_{0}^{1}, \partial_{\infty} G\right)$. But by symmetry, $R\left(a_{0}^{0}, \partial_{\infty} G\right)=R\left(a_{0}^{1}, \partial_{\infty} G\right)$. And the second to last inequality implies $R\left(a_{0}^{0}, \partial_{\infty} G\right)=\infty$.

Corollary 2: In a Gromov hyperbolic graph with a quasipole, the mutually exclusive properties

- $R\left(\omega, \partial_{\infty} G\right)=0$

- $0<R\left(\omega, \partial_{\infty} G\right)<\infty$

- $R\left(\omega, \partial_{\infty} G\right)=\infty$

are coarse geometry invariants.

\section{B. Space localization}

Theorem 11: Given a Gromov hyperbolic graph $G$ with a quasi-pole $\omega$ and a Cantor set Gromov boundary, the effective resistance $R_{G}\left(\omega, v^{i}\right)$, for reference nodes $V_{0}=\{\omega\}$ at the quasi-pole, grows linearly as $d_{G}\left(\omega, v^{i}\right)$,

$$
E\left(\left\|x\left(v^{i}\right)-\hat{x}\left(v^{i}\right)\right\|^{2}\right)=O\left(d\left(\omega, v^{i}\right)\right),
$$

For reference nodes $V_{0}=\partial_{\infty} G$ at the Gromov boundary,

$$
E\left(\left\|x\left(v^{i}\right)-\hat{x}\left(v^{i}\right)\right\|^{2}\right)=O(1)
$$

It may be said that the graph $G$ in Theorem 11 has dimension 2, because the integer dimension of its Cantor boundary is 1. For a 2-dimensional Euclidean lattice, it was shown in [4] that the variance of the error is $O\left(\log d\left(\omega, v^{i}\right)\right)$. The latter and our major result are consistent, as the Euclidean lattice is more wired up than a tree-like graph, so that it is not surprising that the error is smaller in the former.

\section{CONCLUSION}

We have shown that a Gromov hyperbolic graphs with a quasi-pole and a Cantor Gromov boundary is quasi-isometric to a tree and therefore exhibits finite effective resistance to infinity. We have shown that this property has important implications to problems related to propagation and localization problems. However, some coordination problem calls for new challenges, as some standard flocking algorithms [17], [1] enforce a positively curved formation graph, rather than a negatively curved graph such as a Gromov hyperbolic one. This issues remains open for future research.

\section{REFERENCES}

[1] F. Ariaei and E. Jonckheere. Cooperative curvature-driven control of mobile autonomous sensor agent network. In Proceedings of the 46th IEEE Conference on Decision and Control, New Orleans, LA, 2007.

[2] F. Ariaei, M. Lou, E. Jonckeere, B. Krishnamachari, and M. Zuniga. Curvature of sensor network: clustering coefficient. Eurasip, 2006. Submitted.

[3] P. Barooah and J. ao Pedro Hespanha. Estimation on graphs from relative measurements: Distributed algorithms and fundamental limits. IEEE Contr. Syst. Mag., pages 57-74, Aug. 2007.

[4] P. Barooah and J. P. Hespanha. Estimation from relative measurements: Error bounds from electrical analogy. In Proc. of the 2 nd Int. Conf. on Intelligent Sensing and Information Processing, Jan. 2005.

[5] P. Barooah and J. P. Hespanha. Graph effective resistances and distributed control: Spectral properties and applications. In Proc. of the 45th Conf. on Decision and Contr., Dec. 2006.

[6] L. M. Blumenthal. Theory and Applications of Distance Geometry. Oxford at the Clarendon Press, London, 1953.

[7] M. Bonk and O. Schramm. Embeddings of Gromov hyperbolic spaces. Geom. Funct. Analysis, 10:266-306, 2000.

[8] P. L. Bowers. Negatively curved graph and planar metrics with applications to type. Michigan Math. J., 45:31-53, 1998.

[9] M. R. Bridson and A. Haefliger. Metric Spaces of Non-Positive Curvature, volume 319 of A Series of Comprehensive Surveys in Mathematics. Springer, New York, NY, 1999.

[10] J. Cao. Cheeger isoperimetric constants of Gromov-hyperbolic spaces with quasi-poles. Communication in Contemporary Math., 2(4):511533, 2000.

[11] P. de la Harpe. Topics in Geometric Group Theory. Chicago Lectures in Mathematics Series. The University of Chicago Press, 2000.

[12] P. G. Doyle and J. L. Snell. Random walks and electrical networks, 1984.

[13] J.-P. Eckmann and E. Moses. Curvature of co-links uncovers hidden thematic layers in the world wide web. PNAS, 99(9), April 2002.

[14] M. Gromov. Hyperbolic groups. In S. M. Gersten, editor, Essays in Group Theory, volume 8 of Mathematical Sciences Research Institute Publication, pages 75-263. Springer-Verlag, New York, 1987.

[15] M. Gromov. Metric Structures for Riemannian and Non-Riemannian Spaces, volume 152 of Progress in Mathematics. Springer-Verlag, 2001

[16] I. Holopainen, U. Lang, and A. Vhkangas. Dirichlet problem at infinity on Gromov-hyperbolic metric measure spaces. 2005. to appear, available at http://www.helsinki.fi/ iholopai/HLV.pdf.

[17] A. Jadbabaie, J. Lin, and A. S. Morse. Coordination of groups of mobile autonomous agents using nearest neighbor rules. IEEE Transactions on Automatic Control, 48(6):988-1001, 2003.

[18] E. Jonckheere, P. Lohsoonthorn, and F. Bonahon. Scaled Gromov hyperbolic graphs. Journal of Graph Theory, 2007. In press, draft available at http://eudoxus.usc.edu/IW/AIA.html.

[19] E. A. Jonckheere and P. Lohsoonthorn. Geometry of network security. In Proceedings of the American Control Conference (ACC 2004), Boston, MA, June 2004. Paper WeM11.1, Special Session on Control and Estimation Methods in Network Security and Survivability.

[20] E. A. Jonckheere, M. Lou, P. Barooah, and J. P. Hespanha. Effective resistance of Gromov-hyperbolic graphs: application to asymptotic sensor network problems. Technical report, University of California, Santa Barbara, Sept. 2007.

[21] I. Kapovich and N. Benakli. Boundaries of hyperbolic groups. Contemporary Mathematics, 296:39-93, 2002.

[22] M. Kapovich. Hyperbolic Manifolds and Discrete groups, volume 183 of Progress in Mathematics. Birkhauser, Boston, MA, 2001.

[23] P. Lohsoonthorn, E. Jonckheere, and F. Ariaei. Upper bound on scaled Gromov-hyperblic delta. Journal on Applied Mathematics and Computation, 192:191-204, 2007. doi:10.1016/j.amc.2007.03.001. 